\title{
INTRODUCCIÓN. CIENCIA, AGRICULTURA Y SABERES LOCALES EN AMÉRICA LATINA Y EL CARIBE: NUEVAS PERSPECTIVAS
}

\author{
Leida Fernández Prieto (Coord.) \\ Instituto de Historia. CCHS, CSIC
}

Recibido: 8 de enero de 2015. Aceptado: 1 de febrero de 2015.

Cómo citar este artículo/Citation: Fernández Prieto, Leida (coord.) (2015), "Introducción. Ciencia, agricultura y saberes locales en América latina y el Caribe: nuevas perspectivas", Asclepio 67 (1): p075. doi: http://dx.doi.org/10.3989/asclepio.2015.01

\author{
INTRODUCTION. SCIENCE, AGRICULTURE AND LOCAL KNOWLEDGE IN LATIN AMERICA \\ AND THE CARIBBEAN: NEW PERSPECTIVES
}

En las últimas décadas existe un creciente interés entre los historiadores de la ciencia por el estudio de la producción y circulación del conocimiento y las prácticas a diversas escalas (local, regional, nacional, imperial y global). Particularmente, los historiadores de la ciencia cuentan con un campo fértil de indagación dentro de los estudios interesados en el análisis de los espacios donde se produce la ciencia y de los actores involucrados en su quehacer; análisis que subrayan que el conocimiento científico y sus prácticas son locales y que se determinan por medio de un complejo proceso de negociación, lo cual establece una relación mucho más compleja entre centros y periferias (Latour, 1987; Haraway, 1988 (14), pp. 575-599, Ophir y Shapin, 1991, pp. 3-21; Golinski, 1998; Knorr, 1999; Livingstone 2003; Secord, 2004; Raj, 2007).
La historia de la ciencia latinoamericana y caribeña no es una excepción (World History Bulletin, 2006, pp. 1-30; McCook, 2013, pp. 773-776). La imagen de la región como receptora pasiva de saberes y prácticas ha sido ampliamente cuestionada y nuevos estudios demuestran la participación activa en la construcción de una ciencia moderna más abierta que trasciende la tradicional dicotomía entre centros y periferias. No obstante, estos estudios son fragmentarios para la región, mucho más centrados en la historia natural y en la relación triangular ciencia-conocimiento-poder (Cueto y Cañizares-Esguerra, 1999, pp. 46-92; Quintero, 2006, pp. 151-172; 2009a, pp. 14-19 у 2009b, pp. 9-10; Pohl y González, 2009, pp, 7-11). 
El estudio de la producción y circulación de los saberes y prácticas agrícolas en general y científicos en particular, es una asignatura apenas explorada para los historiadores de la ciencia y el medioambiente latinoamericanos y caribeños (Fernández Prieto, 2013, pp. 789-797). Asimismo, se ha atendido mucho al conocimiento tradicional indígena, pero existen otros agentes históricos que ejemplifican el modo en que las comunidades locales organizaron su espacio productor (hacendados, agricultores, compañías trasnacionales, etc.) (Chambers y Gillespie, 2001, pp. 221240; Turnbull, 2003).

Inspirada en estas ausencias, invité a un grupo de especialistas de reconocido prestigio para concebir América latina y el Caribe hispano como un espacio donde se produjo un conocimiento agrícola tropical que interactúo con otras prácticas y saberes desarroIlados a escala global. Igualmente, parece conveniente llevar estos debates al campo de la academia española y dialogar entre un grupo de estudiosos sobre una temática poco explorada para Latinoamérica y el Caribe y con formaciones diversas: historiadores agrarios, ambientales, de la ciencia y geógrafos.

Así, pues, a partir de estudios de casos centrados en el espacio latinoamericano y del Caribe hispano, el monográfico tiene como objetivo principal explorar la construcción y difusión de los saberes agronómicos y las prácticas científicas desarrollados en contextos locales para responder a diversos problemas ecológicos y económicos afrontados por la agricultura en el trópico entre los siglos XVIII y XX, coincidente con la consolidación del modelo agroexportador para la región latinoamericana y caribeña. Se insiste, además, en el papel de la agencia como mediadora para ilustrar el valor del intercambio a diversos niveles (global, regional, local). Los autores atienden a la multiplicidad de agentes socioeconómicos (institucionales y privados) que intervinieron en ello, así como a las diversas conexiones a través de los circuitos del saber. Esto permitirá un análisis comparativo de mayor alcance y contenido en la creación del sistema de conocimientos científicos agrícolas que desdibuje las fronteras entre metrópolis y colonias, local y global.

Asimismo, este monográfico es importante para emprender estudios sobre la participación del mundo colonial y poscolonial en la creación y difusión de saberes y prácticas científico-agrícolas. Para ello se subraya la idea de que cada región tropical productora, incluyendo zonas de América Latina y el Caribe, fueron clave en la construcción, adopción y aplicación de procedimientos científicos. Al mismo tiempo, se enfatiza el valor del intercambio y la interconexión entre estas regiones. Así, se considera una visión más abierta de la participación de la ciencia y la práctica en la agricultura, más allá del debate tradicional de centro versus periferia.

Los autores del monográfico dialogan con diversos enfoques Commodity Histories, Biological Exchange Studies y Knowledge Exchange Studies. Antonio Ortega Santos fue el primer doctorando en historia ambiental que tuvo España. A lo largo de su trayectoria profesional, como profesor de la Universidad de Granada, se ha convertido en uno de los principales interlocutores válidos sobre historia ambiental española y latinoamericana. Ortega en su texto propone emplear el concepto «decolonial» para analizar las continuidades y discontinuidades de los saberes ambientales a los que considera actores protagonistas de procesos históricos como, por ejemplo, epistemicidio; así, explora las consecuencias que supuso la llegada de los saberes europeos sobre las identidades territoriales preexistentes. En su caso, atiende al valor de la agencia de los jesuitas en el proceso de experimentación e hibridación de los saberes con los pueblos nativos de Baja California Sur. El autor señala a los oasis sudcalifornianos como depósitos de saberes ambientales, un término que resulta fructífero para emprender estudios futuros de mayor alcance para la recuperación de saberes y prácticas locales sobre el manejo de los recursos naturales, cuestión trascendental en las agriculturas de plantación tropical conectados al mercado mundial y, por ello, con graves consecuencias medioambientales sobre sus ecosistemas.

Judith Carney es profesora de geografía en la Universidad de California, Los Angeles. Ella es pionera dentro de los estudios que exploran las trazas de la diáspora africana en América y en el mundo Atlántico. Los enfoques Biological Exchanges Studies describen la transferencia de plantas y animales, pero no de saberes. En esa dirección, Carney argumenta la transmisión de saberes llevados por los esclavos a Luisiana, Brasil y México. El texto parte de una versión anterior publicada en inglés, pero desconocida para el mundo hispanohablante. Sobre esa base, la autora introdujo importantes modificaciones y su texto fue traducido por Natalia Santamaría Laorden, talentosa profesora de la Universidad de Ramapo, New Jersey. En su estudio, Carney demuestra el rol fundamental de la agencia de los esclavos en la creación de un sistema de conocimientos en la producción del arroz que sentó las bases de la riqueza de Carolina del Sur, un sistema formado por 
técnicas y habilidades traídas por los esclavos a partir de su previo conocimiento del mundo tropical. La lectura de este texto ofrecerá herramientas metodológicas útiles para llevar a cabo análisis comparativos entre sociedades coloniales de plantación esclavista. Para el caso de Cuba, por ejemplo, este tipo de estudios constituye una asignatura pendiente.

Rafael Marquese es un experimentado historiador agrario y de la historia de la esclavitud en Brasil. En este texto, Marquese analiza la transformación y degradación ambiental provocada por un sistema de administración del paisaje establecido por los propietarios para controlar el trabajo de los esclavos en las plantaciones cafetaleras. En el, recoge las visiones del naturalista francés Auguste de Saint-Hilaire y del agrónomo holandés C.F van Delden Laërne para dibujar el antes y después del boom agroexportador cafetalero sobre los agroecosistemas brasileños. Este texto tuvo una versión inicial publicada en Brasil. Sin embargo, se considera oportuna su publicación en el monográfico por dos razones. Por un lado, para su divulgación entre un público académico hispano-hablante; por otro lado, porque ofrece un enfoque alternativo a los trabajos de Judith Carney, resultando otra mirada valiosa dentro de los estudios de la historia de la esclavitud y de la agencia esclava en la formación de los saberes agrícolas.

Humberto García Muñiz es un reconocido historiador azucarero del Caribe. Su texto destaca la importancia de las revistas azucareras como fuentes por excelencia y poco exploradas para la historia de la industria azucarera tropical. Estas revistas son consideradas como agentes del saber y de prácticas azucareras en formación, centradas en aspectos relacionados con la economía, medioambiente, comercialización, tecnológico y científico de la producción azucarera. Dicho en otras palabras, en sus páginas se trazan las historias de vida y sus relaciones en las diversas fases de la caña de azúcar, desde su cultivo y producción hasta la comercialización, aspectos centrales de los enfoques de las llamadas Commodity History. García Muñiz traza también los recorridos de múltiples hombres de azúcar a los que acuña el sugerente término de "trotamundos del azúcar», entre diversos circuitos del saber. Siguiendo la historia del azúcar, mi trabajo indaga sobre las sugar companys como foco de creación y circulación de saberes a nivel global, pero también dentro de Cuba. En el texto empleo el término de «saberes híbridos» para definir una zona de frontera entre el conocimiento generado por los productores azucareros locales y el nuevo know-how llevado por los norteamericanos a la isla, tras el fin de la guerra hispanoamericana de 1898.

Teresita Levy es una especialista de la historia de la ciencia agrícola de Puerto Rico. Su texto estudia el proceso de extensionismo agrícola, acorde con el modelo de Estados Unidos, como agente y motor del cambio agrícola en Puerto Rico en el contexto del boom agroexportador latinoamericano y caribeño entre finales del siglo XIX y mediados del siglo XX. Ella atiende particularmente a la consolidación de la ciencia dentro del cultivo del tabaco a partir de un marcado interés por parte de los agricultores. Dentro de los estudios denominados Indigenous Knowledge, una de las asignaturas pendientes es precisamente la poca atención prestada a otros agentes locales como, por ejemplo, los agricultores. Ello hace de su texto una importante contribución al dossier.

Por último, Claiton Marcio da Silva es un historiador de la ciencia brasileño. Su trabajo aborda el proceso de negociación entre organismos internacionales y nacionales atravesados por intereses y tensiones imperiales y locales. Para ello, sigue la siempre controversial figura de David Rockefeller y de los actores locales. Concretamente, analiza la puesta en marcha de los proyectos de la agencia estadounidense American International Association for Economic and Social Development (AIA) en las provincias de Sao Paulo y Minas Gerais. La participación de Estados Unidos en América latina y el Caribe se ha estudiado casi siempre desde la mirada del imperialismo económico, científico y ecológico ejercido por éstos sobre la región. Sin embargo, este texto reflexiona sobre la activa participación local en la reorientación y negociación para la implantación de los modelos estadounidenses en Brasil, cuyo resultado no siempre fue exitoso.

En definitiva, los estudios de caso iluminan sobre el papel mediador de diversos agentes socioeconómicos, muchas veces ignorados dentro de la historia de la ciencia. Ello abre líneas de investigación fructíferas para sociedades latinoamericanas y caribeñas.

Deseo agradecer al comité de redacción de la revista, a los evaluadores anónimos y, sobre todo, a los autores, que me han permitido llevar a buen término este proyecto colaborativo y disfrutar de este viaje virtual por la región latinoamericana y caribeña.

\section{AGRADECIMIENTOS}

Este trabajo forma parte del proyecto de investigación HAR2012-37455-C03-01 (MINECO) y el proyecto RYC2009-04030. 


\section{BIBLIOGRAFÍA}

Chambers D. W and R. Gillespie (2001), "Locality in the History of Science. Technoscience, and Indigenous Knowledge," Osiris 15, pp. 221-240.

Cueto, Marcos and Jorge Cañizares-Esguerra (1999), "Latin America." En: DeKosky, Robert and Douglas Allchin (ed.), An Introduction to the History of Science in Non-Western Traditions, Seattle, History of Science Society, pp. 46-92.

Fernández Prieto, Leida (2013), "Islands of knowledge: Science and Agriculture in the History of Latin American and the Caribbean", Isis, 104 (4), pp. 789-797.

Golinski, J. (1998), Making Natural Knowledge: Constructivism and the History of Science. New York, Cambridge University Press.

Haraway, D. (1988), "Situated Knowledge: The Science Question in Feminism as a Site of Discourse on the Privilege of Partial Perspective", Feminist Studies, 14, pp. 575-599.

Knorr Cetina, K. (1999), Epistemic Cultures. How the Sciences Make Knowledge. Cambridge, Massachusetts, London, England, Harvard University Press.

"Latin America in World History", World History Bulletin, vol. XXII, 2, pp. 1-30.

Latour, B. (1987), Science in Action: How to Follow Scientists and Engineers through Society. Milton Keynes, Open University Press.

Livingstone, David N. (2003), Putting Science in its Place: Geographies of Scientific Knowledge. The University of Chicago Press.

McCook, Stuart (2013), "Global Currents in National Histories of Science: the 'Global Turn' and the History of Science in Latin America", Isis, 104 (4), pp. 773-776.
Obregón, Diana (ed.) (2000), Culturas científicas y saberes locales: Asimilación, hibridación y resistencia. Bogotá, Universidad Nacional de Colombia.

Ophir, A. and S. Shapin (1991), "The Place of Knowledge: A Methodological Survey", Science in Context, 4, pp. 3-21.

Pohl, Stefan y Matiana González Silva (2009), "La circulación del conocimiento y las redes del poder", Memoria y Sociedad, 13 (27), pp 7-11.

Quintero Toro, Camilo (2006), “¿En qué anda la historia de la ciencia y el imperialismo? Saberes locales, dinámicas coloniales y el papel de los Estados Unidos en la ciencia del siglo XX", Historia Crítica, 31, pp. 151-172.

Quintero Toro, Camilo (2009a), “Entendiendo los objetos y las mercancías en perspectiva histórica", Historia Crítica, 38, pp. 14-19.

Quintero Toro, Camilo (2009b), “Objetos y mercancías en la historia (II)," Historia Crítica, 39, pp. 9-10.

Raj, K. (2007), Relocating Modern Science. Circulation and the Construction of Knowledge in South Asia and Europe, 1650-1900. Palgrave, Macmilan.

Secord, J. A. (2004), “Knowledge in Transit”, Isis 95, pp. 654-672

Turnbull, David (2003), Masons, Tricksters and Cartographers: Comparative Studies in the Sociology of Scientific and Indigenous Knowledge. London, Routledge. 